\title{
FORMALISER OU SÉCURISER LES DROITS LOCAUX SUR LA TERRE
} ?

Leçons de l'expérience dans la vallée du Sénégal

Patrick d'Aquino, Seydou Camara, Sidy mohamed Seck

Éditions de l'EHESS | «tudes rurales »

$2017 / 1 n^{\circ} 199$ | pages 129 à 148

ISSN 0014-2182

Article disponible en ligne à l'adresse :

https://www.cairn.info/revue-etudes-rurales-2017-1-page-129.htm

\section{Pour citer cet article :}

Patrick d'Aquino et al., « Formaliser ou sécuriser les droits locaux sur la terre ? Leçons de l'expérience dans la vallée du Sénégal », Etudes rurales 2017/1 ( $\mathrm{n}^{\circ} 199$ ), p. 129-148.

Distribution électronique Cairn.info pour Éditions de l'EHESS.

(C) Éditions de l'EHESS. Tous droits réservés pour tous pays.

La reproduction ou représentation de cet article, notamment par photocopie, n'est autorisée que dans les limites des conditions générales d'utilisation du site ou, le cas échéant, des conditions générales de la licence souscrite par votre établissement. Toute autre reproduction ou représentation, en tout ou partie, sous quelque forme et de quelque manière que ce soit, est interdite sauf accord préalable et écrit de l'éditeur, en dehors des cas prévus par la législation en vigueur en France. Il est précisé que son stockage dans une base de données est également interdit. 


\section{études Études rurales \\ rurales 199|2017 \\ La caméra explore les champs}

\section{Formaliser ou sécuriser les droits locaux sur la terre?}

Leçons de l'expérience dans la vallée du Sénégal

Formalizing or Securing Local Rights to Land? Lessons from Experience in the Senegal Valley

Patrick D'Aquino, Seydou Camara et Sidy Mohamed Seck

revues.org

Édition électronique

URL : http://

etudesrurales.revues.org/11590

ISSN : 1777-537X

\section{Éditeur \\ Éditions de l'EHESS}

\author{
Édition imprimée \\ Date de publication : 30 juin 2017 \\ Pagination : 129-148 \\ ISBN : 978-2-7132-2699-1
}

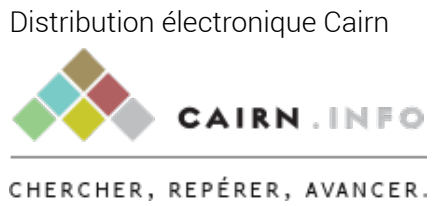

Référence électronique

Patrick D'Aquino, Seydou Camara et Sidy Mohamed Seck, «Formaliser ou sécuriser les droits locaux sur la terre ? ", Études rurales [En ligne], 199 | 2017, mis en ligne le 30 juin 2019, consulté le 18 octobre 2017. URL : http://etudesrurales.revues.org/11590 
Varia 


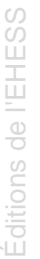

(2)

$\frac{10}{\mathrm{c}}$

$\frac{\sigma}{2}$

ले

焉 
FORMALISER

OU SÉCURISER

LES DROITS LOCAUX

SUR LA TERRE?

\author{
LEÇONS DE L'EXPÉRIENCE \\ DANS LA VALLÉE DU SÉNÉGAL
}

$\mathrm{E}$ $\mathrm{N}$ DEHORS DES PAYS OCCIDENTAUX, beaucoup de populations rurales vivent sur leurs terres sans document écrit attestant de leurs droits. Cependant, les programmes d'investissement, publics ou privés, conduisent souvent à « sécuriser » ces droits locaux, en les répertoriant. Mais leur système complexe est difficilement réductible aux formes usuelles d'enregistrements de droits (cadastre, titrisation...). En effet, ces deux mondes renvoient à des conceptions différentes du rapport à la terre. Il y a d'un côté une vision occidentale basée sur la reconnaissance d'un droit de propriété, assez exclusif, et de l'autre une vision sociétale où les droits sur la terre sont beaucoup moins absolus et partagés en fonction du type d'usage, voire de la saison ou de la ressource considérée.

L'exemple de la partie sénégalaise de la vallée du Sénégal, qui connaît une longue histoire d'implantations de grands périmètres irrigués depuis les années 1960, en fait l'un des plus anciens laboratoires d'expériences pour la prise en compte des droits locaux sur les terres [Seck 2009]. Depuis une trentaine d'années, nous avons participé ${ }^{1}$ ou suivi de
Patrick D’Aquino, Seydou Camara ( + )

et Sidy Mohamed Seck

nombreuses expériences de «sécurisation» dans ce contexte [D'Aquino et al. 1999 et 2002]. Nous proposons, dans un premier temps, une grille d'analyse de cet enjeu de formalisation et ensuite nous exposerons la problématique de sécurisation foncière dans cette partie du Sénégal. Puis, nous appliquerons la grille d'analyse précédemment évoquée à cette région. Enfin, nous confronterons les différences entre les enjeux de formalisation et de sécurisation, en concluant sur la dimension politique et sociétale des choix de sécurisation foncière.

1. Voir I. Faye, A. Benkahla, O. Touré, S. M. Seck et C. O. Ba, 2011, Les acquisitions de terres à grande échelle au Sénégal : description d'un nouveau phénomène, Dakar, Initiative prospective agricole et rurale ; Gret, 2012, Évaluation à mi-parcours du projet d'appui aux communautés rurales de la vallée du fleuve Sénégal (PACR), Saint-Louis/Paris, Gret ; P. D'Aquino, S. M. Seck, et S. Camara, 2011, Recommandations pour le renforcement des capacités et une meilleure appropriation locale des outils de gestion du foncier. Programme MCA d'activité de sécurisation du foncier dans le cadre $d u$ projet d'irrigation et de gestion des ressources en eau de la vallée du Sénégal, MCA Sénégal, Dakar; 2013, Projet de développement inclusif et durable de l'agrobusiness au Sénégal (PDIDAS). Développement de méthodes d'allocation de terrains par les communautés rurales et identification de leurs besoins en assistance technique. Zones de Gandon et du lac de Guiers, Dakar, Cirad, PDIDAS ; 2014, Capitalisation du programme d'appui aux communautés rurales de la vallée du fleuve Sénégal pour la sécurisation foncière (PACR-VFS), Saint-Louis du Sénégal. Voir aussi P. D'Aquino, S. M. Seck, M. Koffii, S. Camara et O. Fedior, 2014, Une démarche d'accompagnement pour mettre en ouvre une gestion territorial et fon-cière décentralisée (<https://agritrop.cirad.fr/575310/1/ document_575310.pdf $>$ ). 
Patrick D'Aquino, Seydou Camara (†) et Sidy Mohamed Seck

\section{Les enjeux d'une formalisation des droits fonciers locaux : sécuriser quoi ?}

De nombreux travaux [Le Bris et al. 1991 ; Lavigne Delville et al. 2002 ; Ribot et Peluso 2003 ; Colin 2008 ; Chouquer 2010 ; Le Roy 2011] ont montré depuis longtemps que derrière le terme de «foncier coutumier» se cachaient des systèmes complexes et évolutifs tant de l'accès aux ressources naturelles que des interactions sociales au sein de la société. Nous pouvons illustrer la façon dont les Sahéliens pensent leurs rapports à la terre sous forme d'un processus chronologique. Un premier groupe social octroie une dimension symbolique à un lieu : en échange de l'utilisation de ses ressources, il s'engage à le préserver. Ce groupe n'est donc pas propriétaire des terres mais, en quelque sorte, le responsable et le garant d'une sage occupation. Il accorde alors à chaque nouvel arrivant une parcelle de «sa » terre qui n'est pas encore occupée. Évidemment, il y a des compensations, des dons réguliers, une sorte d'impôt traditionnel qui repose sur une entente, en partie tacite, sur ce qu'il est bienséant de faire de la part d'une famille qui a été accueillie, en fonction de sa richesse. Cette dernière, une fois installée, occupe les lieux de génération en génération. Ce n'est que lorsqu'elle souhaite agrandir son espace (pour l'installation de jeunes ménages, par exemple) qu'elle demande un nouvel accord à ce chef de terre. Le groupe social permet ainsi le partage de cette terre au gré de l'évolution des relations (alliances, partenariats, assujettissements...) avec d'autres groupes et des nouveaux arrivants. À partir de cette matrice, les règles d'accès à la terre ont évolué au cours des siècles, en s'adaptant et en intégrant les variations du contexte. Cette nature du «droit» sur la terre est la clé de voûte des systèmes fonciers locaux. La notion d'appropriation est progressivement remplacée par celle de lien de responsabilité, entre l'individu et la terre, entre les personnes ellesmêmes et entre les individus et les générations suivantes [Fairhead et Leach 2005]. Des ramifications complexes de droits se sont développées et continuent de se complexifier, à chaque nouvel arrivant, permanent ou saisonnier [Mathieu 1996].

Autrement dit, derrière ce terme de « droit » se cache un objet anthropologique complexe, mais que les cadres réglementaires possibles pour la régulation de l'accès à la terre dénaturent malheureusement. Ainsi l'action publique l'envisage le plus souvent uniquement comme un objet économique, ou bien, dans une perspective plus conciliante, comme la dimension économique d'un objet anthropologique : une "ressource», essentielle dans un processus de développement économique, une base sécurisée d'exploitation durable des ressources. Cependant, les scientifiques spécialistes du Sahel [Behnke et Scoones 1992 ; Bourgeot 1999] insistent sur la posture particulière d'interaction avec l'environnement que contient cet objet anthropologique. En effet, dans les zones arides africaines ces systèmes d'attribution de l'espace débouchent sur des formes originales et flexibles de régulation de l'accès de la majorité des personnes aux rares ressources naturelles. C'est ce que nous appellerons la facette «socio-environnementale» de cet objet anthropologique foncier. 
Fig. 1. Schéma simplifié de la diversité des droits d'accès et d'usage des terres.

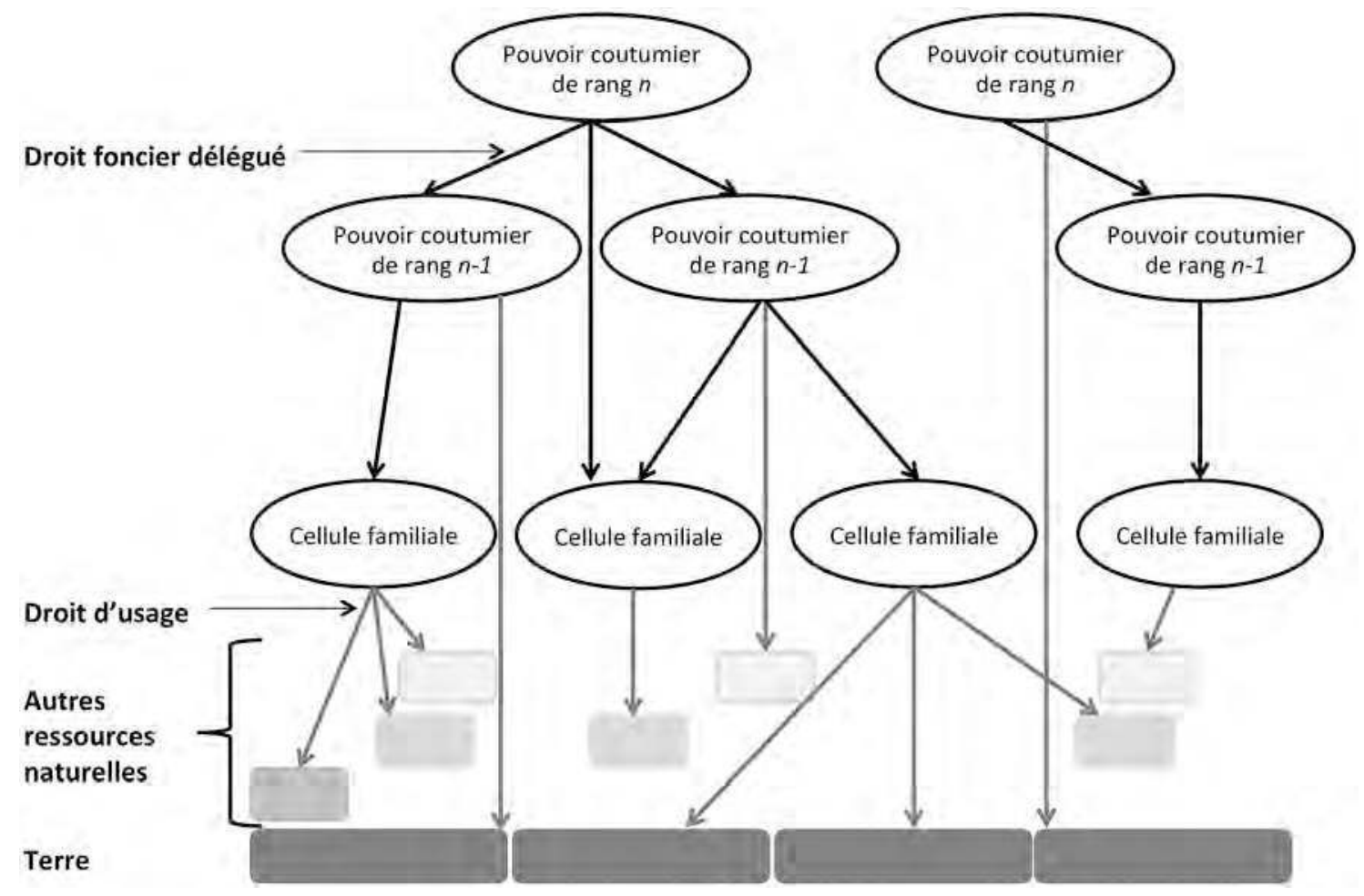


Fig. 2. Distinction simplifiée des différentes facettes du fait social «foncier».

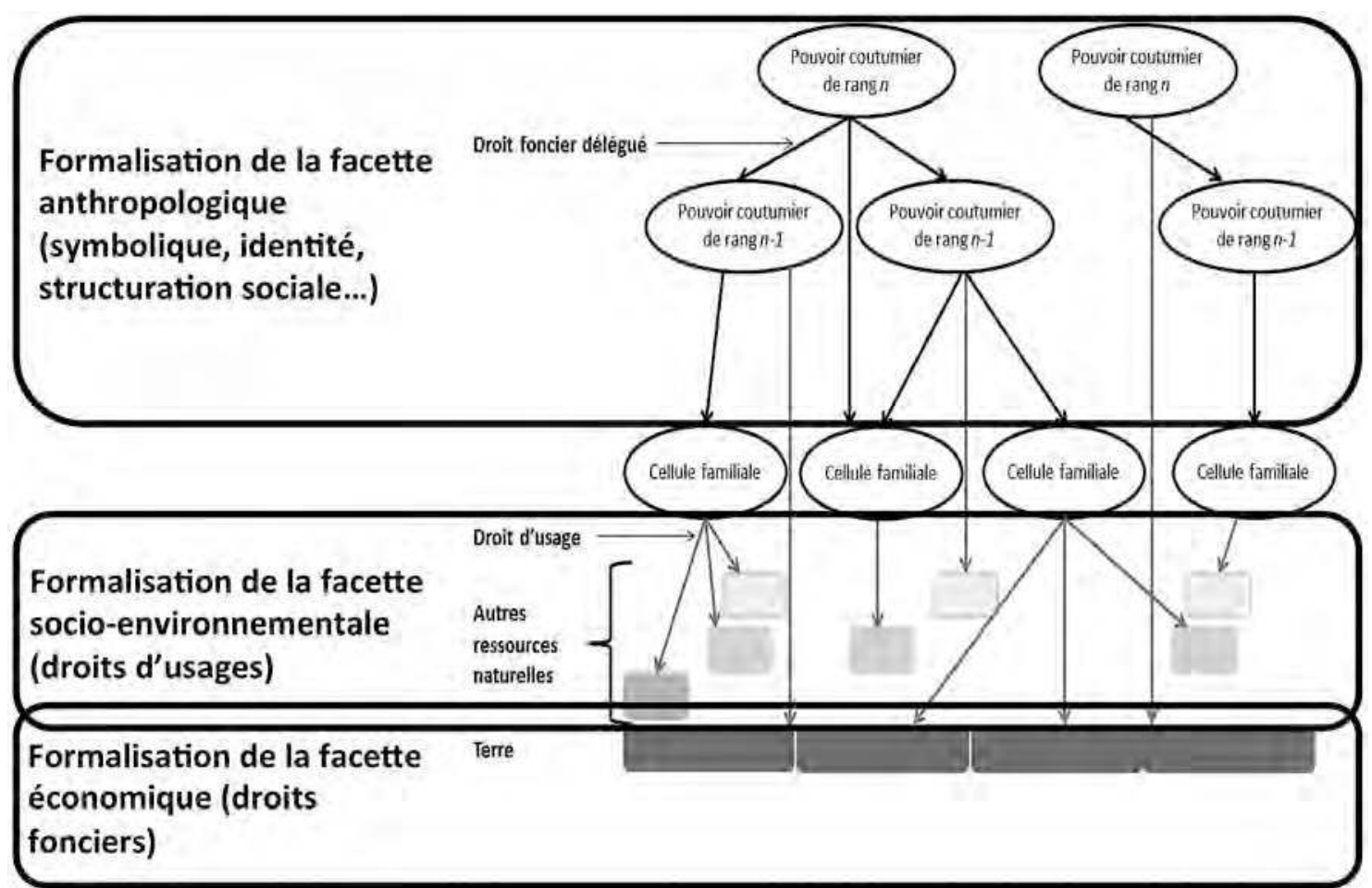


Or, selon la dimension, anthropologique, économique ou socio-environnementale, qui est privilégiée, l'enjeu de formalisation des droits locaux sera interprété différemment, comme nous allons l'illustrer à travers le cas sénégalais. La question de fond ne sera donc pas in fine quel type de formalisation mais quel type de «facette» veut-on, ou doit-on, préserver.

Le fait social foncier comprend trois facettes (fig. 2). La première, anthropologique et présentée ici de façon très simplifiée, est relative aux dimensions identitaires, symboliques, et aux relations sociales, qui déterminent le lien à la terre et, au-delà, structurent plus largement la société et l'expression de ses valeurs. La facette économique, la deuxième, détermine l'utilisation des droits locaux sur la terre comme une ressource du développement économique. Enfin, la troisième - socioenvironnementale - concerne la sécurisation des usages locaux de la terre et des ressources naturelles sous une forme durable et respectueuse de l'environnement. Cette distinction, qui peut paraître abstraite, est apparue cependant très concrètement dans les différentes approches de sécurisation foncière entreprises dans la vallée du Sénégal lors ces dernières décennies.

\section{L'action publique foncière dans la vallée du Sénégal}

Nous allons passer en revue trois mécanismes de formalisation/sécurisation, dont la diversité d'échelles, de portées et d'enjeux permettra de développer une analyse plus générale. Il s'agit de la loi foncière du Sénégal sur le Domaine national, établie depuis 1964 et qui s'applique à l'ensemble du pays, de l'opération plan d'occupation et d'affectation des sols, initiée en 1998 par une équipe de recherche ${ }^{2}$ et qui concerne aujourd'hui la partie sénégalaise du bassin du fleuve Sénégal, et du recueil des droits locaux en préalable à l'attribution foncière de parcelles irriguées, dans le cadre d'un programme du Millenium Challenge Account ${ }^{3}$ lancé en 2010 dans deux zones de la vallée, le delta et la moyenne vallée (département de Podor).

\section{LA LOI SUR LE DOMAINE NATIONAL}

Jusqu'en 1960, coexistaient au Sénégal deux situations foncières: un régime d'immatriculation avec cadastrage dans les quelques zones urbaines et dans le reste du pays des systèmes locaux préexistants à la colonisation [Lericollais et Seck 2002].

Après l'indépendance, la loi sur le Domaine national (LDN) a unifié la situation en attribuant les terres non immatriculées, soit environ $95 \%$ du territoire national, à un domaine détenu par l'État, le Domaine national (fig. 3). Les terres rassemblées dans le Domaine n'appartiennent ni à l'État, ni aux usagers.

2. Équipe Cirad (Centre de coopération internationale en recherche agronomique pour le développement) Isra (Institut sénégalais de recherches agricoles) - Saed (Société publique d'aménagement hydro-agricole de la vallée) du pôle «Systèmes irrigués », Saint-Louis du Sénégal.

3. Le Millenium Challenge Account est un fonds de l'Administration américaine destiné au développement et à la réduction de la pauvreté (<http://www.mcasenegal. org $/ \mathrm{mca} />$ ). 
Fig. 3. Les objectifs de la réforme foncière de 1964 et de la création du Domaine national : supprimer la dualité des régimes fonciers et les domaines fonciers traditionnels.

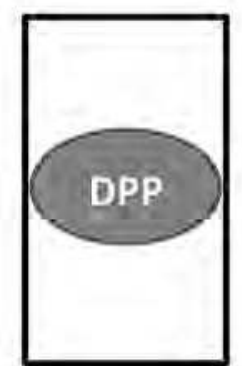

DOMAINE PRIVÉ DES PARTICULIERS (décret juillet 1932, loi 2011-07/03/11)

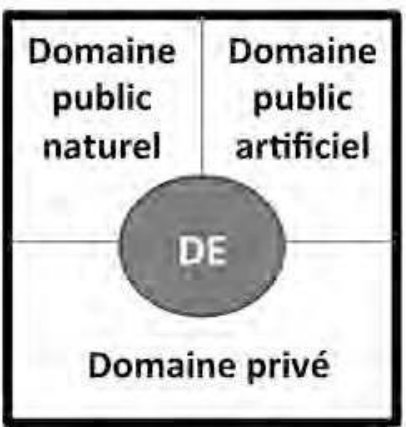

DOMAINE DE L'ÉTAT

(loi 76-77 juillet 1976, décret 81-557 mai 1981 appl. Code Dom. État)

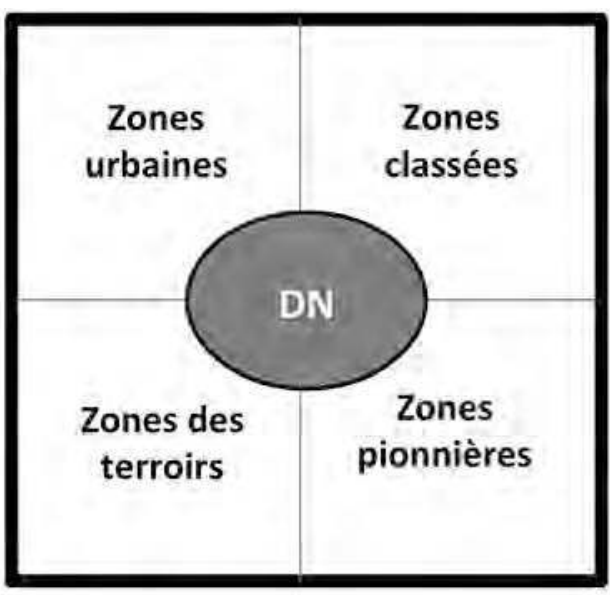

DOMAINE NATIONAL

(loi 64-46 du 30 juillet 1964)

Hétérogène ( 4 zones très différentes) et très vaste (95\% des terres) : "Toutes les terres non classées dans le domaine public, non immatriculées et propriété non transcrite à la date de la Loi » 
Elles restent inaliénables, intransmissibles et incessibles [Le Roy 1985] et sont gérées par les collectivités locales, qui peuvent les affecter à toute personne membre de la collectivité, pour un type d'utilisation considérée comme pertinente.

$\mathrm{Au}$ regard des systèmes fonciers coutumiers, la LDN, toujours en vigueur, a des implications ambiguës. En effet, elle ne reconnaît que ceux qui occupent directement la terre et non leurs responsables coutumiers. Cependant, chaque collectivité locale étant laissée libre de ses choix d'affectation, dans la réalité et depuis des décennies, elle attribue la plupart du temps ces « affectations pour mise en valeur » à ceux qui souhaitent faire reconnaître officiellement leurs droits locaux traditionnels. De toute façon, excepté pour quelques zones d'agriculture intensive, très peu de paysans ont demandé à bénéficier de ce type d'affectation, considérant que la reconnaissance de leurs droits ancestraux était plus sécurisante qu'un «papier » officiel.

L'exploitation agricole entrepreneuriale ${ }^{4}$ a pu s'installer dans ce contexte foncier.

Pour bénéficier d'une sécurisation foncière suffisante, ces entrepreneurs s'arrangent pour obtenir une reconnaissance de leur occupation des terres auprès des deux systèmes de légitimation: la procédure officielle - avec une affectation foncière délivrée par la collectivité locale - et un accord officieux et illégal, résultant d'une négociation avec la population et ses représentants.

Ce n'est donc pas l'absence de sécurité foncière qui freine, au Sénégal, l'investissement des populations dans une agriculture intensive. Pour preuve, des exploitations entrepreneuriales se sont développées dans ce contexte, lorsqu'elles ont stratégiquement pris en compte les droits locaux non officiels. Parmi elles, des exploitations familiales, dont l'histoire se distingue des autres grâce à un capital initial ${ }^{5}$. C'est bien l'absence de moyens financiers qui empêche et a empêché les populations locales d'investir, lorsqu'elles le souhaitaient, dans une mise en valeur plus productive des terres.

Elles ne cherchent à obtenir un «papier» que lorsque les investissements extérieurs se multiplient (espaces irrigués de la vallée du Sénégal, zone maraîchère périurbaine de Dakar). Ainsi dans le delta du Sénégal, le projet de développement inclusif et durable de l'agrobusiness au Sénégal prévoit des aménagements hydro-agricoles réservés aux investisseurs. Jusqu'à cette menace extérieure, les populations n'avaient pas ressenti le besoin de se faire affecter les terres qu'elles occupaient ${ }^{6}$. En 2014, face à ce risque d'accaparement, elles se précipitent auprès de leur collectivité locale pour obtenir les documents officiels, reconnus par le projet et donnant droit à indemnisation. Le paradoxe est alors complet : l'État, associé à ce projet, reconnaît ainsi l'officialisation des droits fonciers locaux via une procédure d'affectation qu'il a mis en place pour effacer ces droits coutumiers. Cette

4. Exploitation agricole avec investissements externes, tournée vers l'intensification et la commercialisation.

5. Voir P. D’Aquino et S. M. Seck, 2014, Agriculteurs $d u$ Sud: des entrepreneurs modernes, Paris, Agence française de développement.

6. Voir P. D’Aquino et al., 2013, Projet de développement inclusif et durable de l'agrobusiness au Sénégal (PDIDAS)... 
Patrick D’Aquino, Seydou Camara (†) et Sidy Mohamed Seck

$\ddot{1} \ddot{8}$

reconnaissance de parcelles foncières au cas par cas, graduelle et non coordonnée, accentue les risques de dérives, car elle n'est encadrée par aucune procédure spécifique de concertation et de contrôle. Par exemple, les populations pastorales enclavées de l'arrière-pays ne participent pas à cette course à la « régularisation » et leurs espaces de vie et de parcours sont alors considérés comme des réserves foncières sur lesquelles les investisseurs vont tenter de s'installer dans le cadre de ce projet.

L'objectif de la LDN n'est pas de sécuriser l'objet anthropologique foncier mais plutôt une exploitation locale des terres ; autrement dit, une sécurisation des usages plutôt que des droits. Ce texte vise aussi à assurer la sécurisation d'une responsabilité collective locale sur la gestion des terres, objectif en grande partie atteint en cinquante années d'application. Cependant, en confiant la gestion de ces affectations aux collectivités locales, donc aux acteurs du système foncier coutumier, la LDN a, en fait, permis à cet objet anthropologique foncier de rester le plus souvent « aux commandes » de la gestion foncière. Ainsi dans la plupart du pays, le «système foncier coutumier » reste aujourd'hui une référence que ce soit dans les critères d'affectation par la collectivité locale, les tractations d'un investisseur pour s'installer ou dans les règles locales d'accès aux terres. Les responsables coutumiers ont donc conservé toute leur légitimité dans ce domaine. Cependant, la procédure d'affectation mise en place a contraint les acteurs locaux à une interprétation très simplifiée des responsabilités foncières traditionnelles, en ne reconnaissant in fine qu'un seul ayant droit, l'affectataire, sur une terre où une diversité de droits d'usages et d'accès s'exprimait (voir fig. 1). De plus, en autorisant des formes individualisées d'affectation de terres, elle a affaibli les notions de responsabilité collective et de non-appropriation individuelle, clé de ces systèmes fonciers, et introduit dans la logique des responsables coutumiers une possibilité de spéculation foncière.

\section{L'opÉRATION POAS DANS LA VALLÉE DU SÉNÉGAL}

En 1998, nous avons participé ${ }^{7}$, avec une collectivité locale de la vallée du Sénégal, à l'expérimentation d'un plan réglementaire de gestion des ressources naturelles. L'objectif est de donner à chaque collectivité locale les capacités d'établir, en concertation avec ses populations, tous types de règles d'occupation des terres lui permettant d'améliorer la sécurisation des différents usages. Il peut s'agir d'identification de zones à réglementation prioritaire pour un usage donné, de la localisation des ressources et infrastructures clés pour chaque usage avec réglementation associée, des règles collectives pour l'ouverture calendaire d'une zone à un usage donné ou encore des modalités d'accès aux résidus de culture pour les troupeaux [D'Aquino et al. 2002].

Ce plan local, bien que dénommé plan d'occupation et d'affectation des sols (POAS), est en fait une version adaptée au contexte national et diverge donc tant dans sa forme que dans son fond de la version française.

7. Programme de recherche d'accompagnement du pôle « Systèmes irrigués », Saint-Louis, Sénégal. 
L'opération POAS se concentre stratégiquement sur les actions et les méthodes qui amènent les différents acteurs à accepter dans les faits les responsabilités et les compétences qui ont été transférées aux collectivités locales en $1996^{8}$. La démarche est ensuite diffusée dans l'ensemble de la vallée, couvrant ainsi en dix ans environ $50000 \mathrm{~km}^{2}$ [D'Aquino et Papazian 2014].

$\mathrm{Au}$ niveau de la sécurisation foncière, l'enjeu de cette approche est double. D'une part, il s'agit d'asseoir la légitimé de la responsabilité des collectivités locales dans leur prise en charge de la gestion de leur territoire, légitimité dont doutent l'encadrement étatique comme les populations. D'autre part, cela doit permettre aux collectivités locales de faire reconnaître officiellement, et donc de protéger, les différents usages existants sur leur territoire. On reste ainsi dans la même logique de sécurisation que la LDN: sécuriser les usages et la gestion collective du foncier. Cependant, l'opération POAS s'est davantage focalisée sur la sécurisation des usages (élevage, agriculture pluviale...) que sur celle des usagers. Par exemple, aucune spécification ne précise quel éleveur a accès aux zones sécurisées d'élevage.

\section{LE RECUEIL DES DROITS LOCAUX EN PRÉALABLE À UNE AFFECTATION DES TERRES}

Beaucoup de travaux ont été réalisés ces dernières années pour rendre opérationnel l'enregistrement des droits locaux [Ouedraogo 2011 ; Stamm 2013 ; CTFD 2015]. Cependant, la superposition de ces derniers sur un même espace pose toujours de nombreux problèmes : l'interprétation peut alors facilement glisser vers une sélection subjective de certains de ces droits lors de l'enregistrement. L'imbrication temporelle et spatiale de cet agencement de droits et droits délégués [Chauveau et al. 2006] dessine une matrice foncière complexe [Leroy et al. 2011], dont la simplification en cadastre foncier ne peut qu'être injuste et inéquitable, en privilégiant certains droits plutôt que d'autres.

Ainsi dans la vallée du Sénégal, le programme intégré de développement de l'agriculture irriguée et de sécurisation des droits du Millenium Challenge Account a dressé à partir de 2011 un diagnostic assez fin des droits locaux, appliqués à des terres occupées traditionnellement et en passe d'être aménagées en périmètres irrigués. Puis, une clé de répartition des terres irriguées distribuant des parcelles aux différents types d'ayant droit (voir les différents acteurs de la fig. 1) a été proposée aux populations. Or, cela a entraîné une opposition violente des responsables locaux. En effet, ces derniers ont considéré qu'un inventaire précis du système foncier ayant été réalisé, il fallait le respecter, distribuer les terres aux responsables fonciers traditionnels qui avaient été répertoriés, donc reconnus, durant l'enquête (voir niveaux $n$ de la fig. 1) et ne pas traiter sur le même plan les droits d'usages et les responsabilités foncières plus fondamentales des chefs de lignage.

Ce cas est révélateur de l'ambiguïté des termes «formalisation des droits » et des enjeux de sécurisation qui s'y rattachent. Deux

8. Loi n ${ }^{\text {9 } 96-06 ~ d u ~} 22$ mars 1996 portant Code des collectivités locales. 
Patrick D’Aquino, Seydou Camara (†) et Sidy Mohamed Seck

traductions «cadastrales », donc sélectives et simplifiées, du système foncier local sont possibles : soit on reconnaît la responsabilité foncière des chefs de lignage, que l'on transforme alors en droit foncier réel ce qui revient alors à dénaturer cette responsabilité collective de gestion en droit foncier individualisé, soit au contraire on préfère attribuer les parcelles à ceux qui y avaient des droits d'usage. Ces deux options sont des interprétations biaisées de la nature des droits et des responsabilités existants sur les terres, menées le plus souvent par des acteurs (bailleurs, équipes de projet, experts, chercheurs...), dont la légitimité à décider d'une telle réorientation de la société est discutable. C'est un choix délicat, inéluctable mais trop souvent implicite, dans toute approche visant à enregistrer, cartographier, formaliser des droits personnalisés et exclusifs sur les terres, dans ces contextes de tenure foncière.

Cependant dans cette vallée, des périmètres irrigués sont régulièrement installés depuis plusieurs décennies sans aboutir à ce type de blocage. La suite du programme MCA permet de l'expliquer. Pour sortir de ce blocage, l'équipe MCA a mis en œuvre une nouvelle approche. Au lieu de s'appuyer sur l'inventaire des droits locaux, un nouveau dialogue a été instauré avec les responsables coutumiers pour définir les clés de répartition des parcelles irriguées, y compris pour la prise en compte des groupes défavorisés. Ainsi, au lieu d'essayer de reconnaître sous forme de droits fonciers réels et individualisés les ayants droit, quels qu'ils soient, cet expert s'est plutôt référé à une reconnaissance de la fonction du système foncier local, c'est-à-dire de sa responsabilité collective dans la gestion et donc ici dans la réaffectation des terres. Autrement dit, il reconnaît les pratiques et les règles existantes ${ }^{9}$ plutôt que d'imposer ex nihilo une nouvelle forme de relation à la terre et un droit individualisé.

L'enjeu de l'intervention du sociologue est de faire évoluer la situation foncière locale, donc les critères de répartition des terres irriguées au sein de la communauté, pour favoriser l'accès à la terre des jeunes, des femmes et des autres groupes défavorisés. Ces critères ont été proposés, avec argumentation, à la communauté locale qui les a intégrés dans la répartition des parcelles ${ }^{10}$ [MCA 2012]. Nous n'avons évidemment pas la naïveté de croire que cette évolution des clés de répartition a dépossédé les notables de leurs droits sur les terres. En revanche, elle a ouvert l'accès aux terres irriguées à des personnes qui en étaient privées jusque-là. Elle a permis la prise en compte, dans le processus local de gestion collective, de la reconnaissance communautaire. On peut rapprocher cette pratique de la façon dont la LDN est interprétée sur le terrain: certes les droits locaux ne sont pas reconnus officiellement, mais les acteurs ayant une responsabilité de gestion dans le système foncier local (chefs de terres, lignages...) sont en réalité impliqués dans toute décision concernant l'attribution des terres.

9. Voir la pratique existante de gestion communautaire des terres (fig. 1 et 2) dont les règles diffèrent de celles d'une individualisation des droits fonciers.

10. Voir MCA, 2012, Syllabus sur le Ngalenka. Projet MCA-Sénégal. Dakar, MCA (<http://agritrop.cirad.fr/ $581843 />)$. 


\section{Quels enjeux de sécurisation}

\section{des droits fonciers locaux au Sénégal ?}

Il y a, d'un côté, la formalisation du système foncier local par une description fine permettant ensuite de répartir des ayants droit individualisés lors d'un cadastrage et, de l'autre, le fait de considérer ce système comme une « boîte noire » complexe, que l'on ne cherche pas à transcrire sous peine de le dénaturer, mais auquel on reconnaît une responsabilité collective dans la gestion du foncier. Autrement dit, la sécurisation de droits réels individualisés ou la sécurisation du système de gestion des droits. Les deux options présentent des risques : dans le premier cas, la responsabilité de l'équité est transférée à l'intervenant décidant des affectations, dans le second elle relève de la collectivité locale gestionnaire des droits.

Or, la sécurisation des droits individualisés est revendiquée pour répondre à deux besoins presque opposés. Il y a, d'une part, la demande de stabilité des institutions internationales pour les investissements nécessaires au développement d'une production intensive et durable et, de l'autre, l'attente des populations des revenus tirés de l'exploitation future des terres, avec des positions divergentes sur les légitimes bénéficiaires (Les responsables fonciers traditionnels? Tous les ayants droit, y compris d'usage? La communauté dans son ensemble?).

Si l'on revient aux figures 1 et 2 , on constate que ces deux revendications consistent à traduire le système foncier uniquement à travers sa facette économique - la terre comme ressource et le droit foncier local comme un droit d'exploitation économique de cette ressource - avec la difficulté liée à la sélection de droits exclusifs à enregistrer à partir d'une interprétation de l'objet anthropologique foncier. Cette distribution de droits individualisés est souvent considérée comme une base nécessaire à l'investissement productif. Or, en ce qui concerne le Sénégal, cette analyse est facilement réfutable car le système foncier local n'est pas fragilisé par des pressions extérieures, les populations locales se considérant suffisamment reconnues dans leurs droits pour ne pas recourir à un dispositif de formalisation. Nous avons aussi constaté que des investisseurs étrangers se sont contentés de ce type de reconnaissance locale pour investir dans la vallée du Sénégal depuis des décennies. Il n'est donc pas déraisonnable d'envisager dans ce pays d'autres voies de sécurisation foncière de l'investissement que l'appropriation privative individualisée. La nécessité d'un droit foncier individualisé comme unique possibilité de garantie pour un soutien bancaire ne tient pas non plus: d'autres solutions sont plus adaptées dans de nombreuses situations agraires dans le monde. Au Sénégal, spécifiquement, les banquiers de proximité considèrent qu'une assurance agricole individualisée constitue une garantie bancaire plus sûre qu'une hypothèque foncière. Même une agriculture de firme a d'autres moyens de garantie à sa disposition pour bénéficier de soutiens financiers : la vallée se couvre d'exploitations privées, en particulier étrangères. Le contexte foncier n'est donc pas un obstacle à ces lourds, et réfléchis, investissements.

Enfin, à l'échelle nationale comme locale, d'autres acteurs (dont l'organisation non gouvernementale Enda-Pronat) soutiennent une 
Patrick D’Aquino, Seydou Camara (†) et Sidy Mohamed Seck

autre sécurisation foncière en préconisant de garder la responsabilité collective sur les terres de façon à préserver le patrimoine foncier et environnemental pour les générations futures. Derrière cette position transparaît la préservation du lien identitaire et symbolique avec la terre, l'essence fondamentale de ces systèmes fonciers, que l'on retrouve dans les débats paysans ${ }^{11}$.

Au Sénégal, les stratégies de sécurisation foncière développées par les investisseurs implantés depuis des décennies montrent que la forme la plus sûre d'occupation foncière est celle qui obtient une double légitimité : celle des populations locales, au moyen d'une contractualisation officieuse entre l'investisseur et les populations locales, couplée à celle, officielle, de l'État ou des collectivités locales. Or, le renforcement de la sécurisation ne peut passer que par la reconnaissance mutuelle de ces deux types de légitimité. Cependant, poursuivre vers cette option signifierait renoncer au régime de propriété privée, afin de conserver une certaine responsabilité collective de gestion de la terre, avec en perspective la création de droits délégués (bail, location, fermage, métayage...).

De plus, dans un contexte sahélien marqué par une très forte incertitude environnementale, cette articulation souple de droits d'accès et d'usage des ressources naturelles peut être fondamentale à préserver.

Mais pour traduire aujourd'hui cette flexibilité ancestrale, on se retrouve devant les mêmes défis que pour l'interprétation des règles foncières : d'un côté, la structuration sectorielle des politiques publiques gêne cette appréhension globale et, de l'autre, la rigidité des outils d'aménagement du territoire permettent mal cette flexibilité. Or, là aussi l'expérience sénégalaise montre qu'une flexibilité opérationnelle est possible ${ }^{12}$ : plutôt que d'imposer un arsenal de réglementations emboîtées et figées, il vaut mieux identifier et sécuriser des instances collectives de régulation à différentes échelles, qui auront la possibilité de produire, mais surtout de faire évoluer de façon flexible, la distribution des droits d'occupation et d'usage de l'espace.

\section{Formaliser ou sécuriser?}

La distribution de droits individualisés permet de sécuriser le bénéfice d'une personne concernant une terre en particulier, y compris lors de sa vente à un investisseur. Quant à la reconnaissance d'un droit local d'administration des terres, elle sécurise la préservation des terres agricoles pour la communauté locale par des règles contrôlant les transferts de droits d'usage. Il ne s'agit pas de considérer l'une ou l'autre de ces options comme « la » bonne solution mais de souligner le fait que selon ce que l'on formalise, on ne sécurise pas la même chose. Le terme de formalisation contient l'idée de transformation en un système précis et fixé d'une pratique ou d'une règle peu claire. Faut-il clarifier le «flou» des droits fonciers locaux ?

11. Voir P. D’Aquino, 2014, Foncier rural au Sénégal : paroles d'experts paysans. Extraits d'ateliers paysans de simulation. Projet de renforcement des capacités de prospective participative en réforme foncière des organisations de la société civile. Dakar et Paris, EndaPronat, comité technique «Foncier et développement».

12. Voir note 1. 
Le premier constat que nous pouvons tirer de l'expérience sénégalaise est que réduire la complexité des faisceaux de droit existants, en appliquant une clé de redistribution sera toujours subjective, donc politique. Ce n'est donc pas une méthode de formalisation qu'il s'agit de sélectionner, mais un choix de société à opérer ${ }^{13}$. Notre première remarque est donc qu'il est impératif que l'institution en charge de ce choix ainsi que le processus de dialogue local mis en œuvre pour l'effectuer bénéficient d'une légitimité sociale comme politique. La deuxième concerne la place importante accordée à la cartographie foncière dans la plupart des interventions : la considérer comme l'aboutissement inéluctable de toute sécurisation nous paraît discutable. Certes, il existe des approches originales qui tentent de concilier individualisation cartographiée des droits d'usage et administration foncière locale, mais reste cependant posée la question de la pertinence d'une cartographie foncière, quelle qu'elle soit, pour initier une « sécurisation » des «droits locaux », termes très éloignés de la nature réelle des systèmes fonciers locaux (voir supra).

En effet, les spécialistes de l'agropastoralisme des zones sèches ont depuis longtemps montré que ce qui apparaissait comme flou était, en réalité, le caractère flexible du système de règles d'accès aux ressources naturelles, autrement dit une souplesse organisée permettant de s'adapter à la variabilité et à l'incertitude constante et extrême du milieu [Scoones 1994 ; Mehta et Leach 1999 ; Colin 2008 ; Leroy 2011]. Face à la tentation de formalisation, une question se pose, bien au-delà du cas sénégalais: est-ce qu'il ne serait pas utile de préserver cette flexibilité, ce «flou», dans un monde contemporain de plus en plus marqué par l'incertitude climatique comme économique ? Dans cette perspective, n'est-il pas contre-productif de baser une reconnaissance des droits locaux sur une cartographie et sur une formalisation, figeant ainsi des règles souples ? Il nous paraît donc plus pertinent de remplacer l'enjeu de formalisation par celui de sécurisation. Ainsi l'analyse scientifique fine de la complexité des droits coutumiers, certes d'une importance capitale pour la compréhension de ces systèmes et à laquelle nous avons participé, est discutable en tant que méthode de reconnaissance formelle de droits. En effet, cette opération de traduction serait politique et donc devrait être traitée comme telle et relever non pas de la responsabilité des experts et scientifiques mais des institutions légitimes pour effectuer ces choix. De plus, au regard de la nature même de ces droits coutumiers, nous considérons que cette traduction de la connaissance en normes est loin d'être le plus sûr moyen de sécuriser les responsabilités locales sur les terres.

Autrement dit, il ne peut y avoir de proposition scientifique pour un mécanisme de transcription de l'objet anthropologique « système foncier local » en droits positifs. La légitimité de l'expertise scientifique se restreint finalement à deux domaines.

13. Voir V. Basserie et P. D’Aquino, 2011, Sécurisation et régulation foncières : des enjeux aux outils. Quelques obstacles à la cohérence des politiques, fiches pédagogiques, CTFD (<http://www.agter.org/bdf/_docs/ctf_basserie_ daquino_securisation_regulation_fonciere_fr.pdf $>$ ). 
Patrick D'Aquino, Seydou Camara (†) et Sidy Mohamed Seck

Le premier se situe, en amont, avec une description la plus fine possible des droits existants, mais dont l'intérêt en termes de sécurisation réside dans l'identification des institutions locales régulatrices du foncier et de leur mode de fonctionnement (et non la formalisation de droits réels individualisés sur les terres) et dans une analyse de l'impact des différents choix. Le second domaine se trouve en aval, après le travail politique de choix des droits à transcrire, dans la mise au point du processus de leur enregistrement.

Ramener l'enjeu de sécurisation foncière à une question politique met alors en exergue une question de fond qui traverse les débats fonciers au Sénégal : la probable tension entre l'aspiration historique de l'intelligentsia à une société progressiste et humaniste - qui s'est profondément inscrite dans l'identité sénégalaise - et la préservation des valeurs culturelles de partage et d'adaptation à l'environnement d'une grande partie de la population rurale. Alors que ces deux aspirations pouvaient cohabiter dans la situation foncière permise par la LDN, qui facilitait la sécurisation foncière d'une exploitation économique des ressources tout en reconnaissant aux populations une gestion locale de leurs droits ancestraux sur les terres, elles semblent se trouver en concurrence face aux évolutions actuelles de ce pays.

Plus fondamentalement, cette crispation que l'on sent actuellement dans les débats au Sénégal lorsque des acteurs de la société civile ${ }^{14}$ revendiquent une forte délégation au local de responsabilités sur la gestion foncière et territoriale interroge la place et le pouvoir de l'État, son rapport et son autorité sur le territoire. En effet, le type de reconnaissance du local que cela impliquerait peut s'interpréter comme un autre type de société que la formule étatique actuelle : un «État de droits », garant des droits locaux et de leur application équitable et durable, plutôt qu'un «État de droit », qui impose ses propres droits ${ }^{15}$. Ainsi lorsque l'on soumet à des paysans sénégalais la question de l'héritage des droits sur les terres, ils suggèrent que plutôt que de s'appuyer sur une règle d'héritage applicable partout et pour tous, il vaudrait mieux laisser chaque collectif local de responsables décider au cas par cas de l'héritier le plus à même de mettre en valeur les terres, enjeu prioritaire pour la société... On voit que la prise en compte du système foncier local va alors bien plus loin qu'une simple attribution de droits fonciers et interroge à nouveau le mode d'organisation de la société.

\section{Conclusion}

Il y a moins d'opposition qu'il n'y paraît entre une société libérale, préconisant les mêmes droits d'accès à la terre pour tous, et une société qui met en avant une adaptation des droits de chacun à une préservation du patrimoine naturel et, en particulier, de la terre. En effet, ce type de fonctionnement n'est pas si étonnant et improbable car il est déjà en grande partie en place dans le cadre légal sénégalais (voir $L D N$ ) et il suffirait de peu pour légitimer et rendre plus effective son application.

14. <http://www.inter-reseaux.org/publications/revuegrain-de-sel/57-foncier-innover-ensemble/article/quandla-societe-civile-s-allie-au ?lang=fr.>.

15. Communication personnelle de Philippe Karpe. 
L'obstacle principal est, en fait, qu'il semble représenter un choix alternatif de société, une façon différente de considérer la place de l'Homme, de l'État et de la nature, comme la logique de leurs interactions. La question de sécurisation foncière dans ce contexte amène ainsi à une question de société qui interpelle bien au-delà du Sénégal. En effet, les modes alternatifs de gestion des terres qui y transparaissent y sont suffisamment opérationnels pour envisager raisonnablement une forme de gouvernance des ressources naturelles qui ne soit pas basée sur une relation personnalisée d'appropriation, mais plutôt sur une responsabilisation accrue de la société sur la préservation à long terme de son environnement. Responsabilisation plutôt qu'appropriation... Même si les fondations théoriques et méthodologiques d'un tel choix peuvent exister, encore faut-il que notre société parvienne à les accepter.

\section{Bibliographie}

Behnke, Roy et lan Scoones - 1992, Repenser l'écologie des parcours : implications pour la gestion des terres de parcours en Afrique. Londres, IIED.

Bourgeot, André (dir.) - 1999, Horizons nomades en Afrique sahélienne: sociétés, développement et démocratie. Paris, Karthala.

Chauveau, Jean-Pierre, Jean-Philippe Colin, JeanPierre Jacob, Philippe Lavigne Delville et PierreYves Le Meur - 2006, Mode d'accès à la terre, marchés fonciers, gouvernance et politiques foncières en Afrique de l'Ouest. Londres, IIED.

Chouquer, Gérard - 2010, "Enjeux fonciers. Troisième partie: thèmes transversaux », Études rurales $186: 251-272$.

Colin, Jean-Philippe - 2008, " Disentangling IntraKinship Property Rights in Land: a Contribution of Economic Ethnography to Land Economics in Africa », Journal of Institutional Economics 4 (2) : 211-254.

Comité technique "Foncier et développement" (CTFD) - 2015, La formalisation des droits sur la terre dans les pays du sud. Dépasser les controverses et alimenter les stratégies. Paris, ministère des Affaires étrangères et du Développement international/Agence française de développement.

D'Aquino, Patrick, Sidy Mohamed Seck, JeanFrançois Bélières, Michel Passouant et El Hadj Malick Sarr - 1999, "Irrigation et développement régional: dix ans d'actions sur le fleuve Sénégal pour une planification régionale et décentralisée ", Cahiers de la Recherche-Développement 45 : 77-84. D'Aquino, Patrick, Sidy Mohamed Seck et Seydou Camara - 2002, "L'acteur avant l'expert: vers des systèmes $d$ 'information territoriaux endogènes. Une expérience au Sénégal », Natures, Sciences, Sociétés 10 (4) : 20-30.

D'Aquino, Patrick et Hermine Papazian - 2014, «An Inclusionary Strategy Reaching Empowering Outcomes Ten Years after a Two-Year Participatory Land Uses management », Environmental Management and Sustainable Development 3 (2) (<http:// dx.doi.org/10.5296/emsd.v3i2.6595>).

Fairhead, James et Melissa Leach - 2005, "The Centrality of the Social in African Farming », IDS Bulletin 36 (2) : 86-90.

Lavigne Delville Philippe, Camilla Toulmin, JeanPhilippe Colin et Jean-Pierre Chauveau - 2002, Sécurisation des droits fonciers délégués en Afrique de l'Ouest. Londres, IIED.

Le Bris, Étienne, Étienne Le Roy et Paul Mathieu (dir.) - 1991, L'appropriation de la terre en Afrique noire. Manuel d'analyse de décision et de gestion foncières. Paris, Karthala.

Lericollais, André et Sidy Mohamed Seck - 2002, "Patrimoines anciens et territoires en gestation dans la région riveraine du fleuve Sénégal » in J. Boutrais, M.-C. Cormier-Salem, D. Juhé-Beaulaton et B. Roussel (dir.), Patrimonialiser la nature tropicale. Dynamiques 
Patrick D'Aquino, Seydou Camara (†) et Sidy Mohamed Seck

$\ddot{146} \quad$ locales, enjeux internationaux. Paris, IRD/MNHN : 283-300.

Le Roy, Étienne - 1985, "La loi sur le domaine national (du Sénégal) a vingt ans. Joyeux anniversaire?", Mondes en développement 13 (52) : 667685. - 2011, La terre de l'autre. Une anthropologie des régimes $d$ 'appropriation foncière. Paris, Maison des sciences de I'homme/LGDJ.

Mathieu, Paul - 1996, "Pratiques informelles, gestion de la confusion et invention du foncier en Afrique ", in G. de Villers (dir.), Phénomènes informels et dynamiques culturelles en Afrique. Paris, L'Harmattan : 64-87.

Mehta, Lyla, Melissa Leach et Ian Scoones - 1999, Exploring Understandings of Institutions and Uncertainty: New Directions in Natural Resource Management. IDS Discussion Paper 372, Institute of Development Studies, Brighton.

\section{Résumé}

Patrick D’Aquino, Seydou Camara ( $\dagger$ ) et Sidy Mohamed Seck, Formaliser ou sécuriser les droits locaux sur la terre ? Leçons de l'expérience dans la vallée du Sénégal La notion de droits fonciers recouvre des réalités bien différentes selon l'interlocuteur et le contexte, en particulier dans les situations au Sud marquées par une appréhension du foncier bien différente des conceptions occidentales. Cet article montre qu'il ne peut y avoir de traduction objective de ces droits et que, selon la facette (anthropologique, économique et environnementale) que l'on privilégie, la nature des droits qui seront reconnus diffèrera. La démonstration s'appuie sur les mécanismes de formalisation de droits mis en œuvre dans la partie sénégalaise de la vallée du Sénégal durant les deux dernières décennies. Elle y argumente que l'option pour la reconnaissance des formes locales de régulation (cf. la facette anthropologique) plutôt que des droits conduit à envisager de nouvelles formes de gouvernance des ressources naturelles qui ne soit pas basée sur une relation personnalisée d'appropriation, mais plutôt sur une responsabilisation accrue de la société sur la préservation à long terme de son environnement.

\section{Mots clés}

Sahel, Sénégal, vallée du Sénégal, accaparement, collectivités locales, droits coutumiers, droits d'usage, Domaine national, réforme foncière, sécurisation des droits, terres agricoles
Ouedraogo, Hubert - 2011, " De la connaissance à la reconnaissance des droits fonciers africains endogènes ", Études rurales 187 (1) : 79-93.

Ribot, Jesse C. et Nancy Lee Peluso - 2003, "A Theory of Access », Rural Sociology 68 (2) : 153-181. Scoones, Ian - 1994, Living With Uncertainty: New Directions in Pastoral Development in Africa. London, International Institute for Environment and Development. Seck, Sidy Mohamed - 2009, "Changements institutionnels et difficultés de développement hydroagricole dans le Delta du fleuve Sénégal : nouvelles dynamiques et recompositions autour de l'irrigation », in E. Dansero, E. Luzzati et S. M. Seck (dir.) Organisations paysannes et développement local. Leçons à partir du cas du delta du fleuve Sénégal. Paris, L'Harmattan Italia : 21-52.

Stamm, Volker - 2013, "Formaliser les pratiques coutumières. Europe médiévale, Afrique coloniale et contemporaine », Études rurales 191 (1) : 169-189.

\begin{abstract}
Patrick D'Aquino, Seïdou Camara $(\dagger)$ and Sidy Mohamed Seck, Formalizing or Securing Local Rights to Land? Lessons from experience in the Senegal Valley The notion of land rights means very different things depending on who is speaking and the context, particularly in situations in the Global South where the understanding of land can be very different than in the West. This article shows that an objective translation of these rights is not possible and that, depending on the chosen angle of approach (anthropological, economic or environmental), the nature of such rights is recognized differently. The example presented is based on the tools implemented to formalize land rights over the last two decades in the Senegalese part of the Senegal Valley. It argues that opting to recognize local forms of regulation (i.e., an anthropological approach) rather than rights leads to envisaging new types of governance for natural resources that are not based on a personalized relationship of appropriation but rather on raising awareness within society regarding the long-term protection of the environment.
\end{abstract}

\section{Keywords}

Sahel, Senegal, Senegal Valley, land grabbing, local municipalities, customary rights, right of user, public land, land reform, securing rights, agricultural land 
Hommage à Seydou Camara (1960-2016)

Seydou Camara, s'en est allé subitement, dans la force de l'âge, le 25 février 2016 à Saint-Louis (Sénégal), où il était né le 2 février 1960. Seydou était un extraordinaire sociologue de terrain, totalement engagé depuis plus de 30 ans dans la reconnaissance des modes d'organisation des populations locales dans la mise en valeur des terres de la vallée du Sénégal. Après un troisième cycle en sociologie à l'Université de Saint-Louis, achevé en 1986, il a occupé de 1990 à 2007 plusieurs postes à responsabilités à la Société d'aménagement de la vallée (Saed), avant de mettre son expertise, jusqu'à son décès, au service de différents programmes d'appui à l'agriculture irriguée dans la vallée. Loin des cadrages théoriques, trop impliqué à façonner sur le terrain des arrangements sociaux pour avoir le loisir de publier, il a ainsi été, dans l'ombre du terrain mais aussi de son humilité remarquable, celui qui a constamment innové et fait progresser les modes de gestion des eaux et des terres irriguées dans la partie sénégalaise de la vallée du Sénégal, au cours des trois dernières décennies. Avec ce présent article et les échanges avec les deux autres auteurs qu'il avait entraînés, il avait enfin eu l'opportunité de voir intégrer et formaliser cette intuition qui l'a motivé tout au long de sa carrière professionnelle, celle de s'appuyer sur les ententes sociales plutôt que sur les règles formelles. Malheureusement, il n'aura pas eu la satisfaction de voir ses idées enfin reconnues et publiées. La vallée du Sénégal ne sera plus jamais la même, pour tous ceux qui y œuvrent pour le développement et la reconnaissance des populations. Au revoir, très cher ami.

P. D'Aquino 


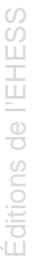

(2)

$\frac{10}{\mathrm{c}}$

$\frac{\sigma}{2}$

ले

焉 